Editorial

\section{Pediatric surgical activity}

\section{Editorial}

In the past 30 years pediatric surgeons and pediatric surgical research where focused on "new" diseases that storically were treated by general surgeons.

From a strictly research point of view, pediatric surgeon have to study how can they improve health in those patients that have surgical problems; storically pediatric surgeons have to know all gastrointestinal disorders, genitourinary disorder, and respiratory disorders. Many of pediatric surgeon activity are about congenital malformations.

In small or very small babies, necrotizing enterocolitis is one of the most important diseases. In the past few years, the percentage of died babies decreased also thanks to the basic research. Our group was one of first that described the role of Probiotics to prevent clinical deterioration in patients with necrotizing enterocolitis. However, not only surgical skills are essential for these patients.

So far pediatric surgeons had the opportunity to study new aspects of different pathologies, and recently many researchers on these fields are changing. Our Team storically was a referral Centre for pediatric urology, with great experience about urological disease affecting kidney, ureters, bladder and urethra. Many studies were focused about vesicoureteral reflux, uretero-pelvic-junction obstruction and hypospdias.

When, more than 15 years ago, a new aspect of pediatric urology opened new research field such as pediatric andrology, our team organized multiple studies; we started to study undescended testes and varicocele that are at present, the two most important disease affecting fertility potential in adulthood; with great results our Team is one of the most important andrological research group worldwide with many papers, congress presentation and surgical trials.

But pediatric surgery is not only "surgery" and really our main point is to preserve child health. For this reason our team organized o multidisciplinary Team to study and preserve fertility also in pediatric age.
Volume I Issue I - 2017

Francesco Saverio Camoglio

Pediatric Surgery Unit, University of Verona, Italy

Correspondence: Francesco Saverio Camoglio, Pediatric Surgery Unit, Woman \& Child Hospital, University of Verona, Verona, Italy, Email francesco.camoglio@univr.it

Received: July 12, 2017 | Published: July 21, 2017

So, as in adulthood, many disease such as obesity, can affect fertility; recently we have the role of "national coordinator" for adolescent bariatric procedures registry, where all bariatric Centre have to add clinical aspect of these adolescent. Starting from these results we opened also a cryopreservation centre for testicular tissue with other teams to offer for those children with testicular problems (oncological disease or trauma) to preserve their gonadal tissue for future fertility.

Our recent studies demonstrate that this field is important especially for parents that could have some positive news about the future of their child. Respect to other specialist, pediatric surgeon has an important role for the future of their patients; research should focus on diagnosis, management, treatment and follow up. All pediatric surgical team should have, for this reason, the interest to better understand these new aspects of surgical diseases.

\section{Acknowledgements}

None.

\section{Conflict of interest}

The author declares no conflict of interest. 